Accepted refereed manuscript of:

O'Connor DB, Green JA, Ferguson E, O'Carroll R \& O'Connor RC (2018) Effects of childhood trauma on cortisol levels in suicide attempters and ideators,

Psychoneuroendocrinology, 88, pp. 9-16.

DOI: 10.1016/i.psyneuen.2017.11.004

(C) 2017, Elsevier. Licensed under the Creative Commons Attribution-NonCommercialNoDerivatives 4.0 International http://creativecommons.org/licenses/by-nc-nd/4.0/ 


\section{Effects of childhood trauma on cortisol levels in suicide attempters and ideators}

Daryl B. O'Connor, $\mathrm{PhD}^{1 *}$, Jessica A. Green, $\mathrm{MSc}^{1}$, Eamonn Ferguson, $\mathrm{PhD}^{2,}$, Ronan E. O'Carroll, $\mathrm{PhD}^{3}$, \& Rory C. O'Connor, $\mathrm{PhD}^{4}$

${ }^{1}$ School of Psychology, University of Leeds, Leeds UK ${ }^{2}$ School of Psychology, University of Nottingham, Nottingham, UK

${ }^{3}$ Division of Psychology, University of Stirling, Stirling, UK

${ }^{4}$ Suicidal Behavior Research Laboratory, Institute of Health \& Wellbeing, University of Glasgow, Glasgow, UK

Running head: Childhood trauma, cortisol and suicide

Correspondence to:

Daryl B. O’Connor

School of Psychology

University of Leeds,

Leeds, UK

e: d.b.oconnor@leeds.ac.uk

t: ++44 1133435727

Accepted for publication in Psychoneuroendocrinology published by Elsevier. 


\title{
Effects of childhood trauma on cortisol levels in suicide attempters and ideators
}

\begin{abstract}
Objectives: Suicide is a global health issue. Dysregulated hypothalamic-pituitary-adrenal (HPA) axis activity, as measured by cortisol levels, has been identified as one potential risk factor for suicide. Recent evidence has indicated that blunted cortisol reactivity to stress is associated with suicidal behavior. The current study investigated whether childhood trauma was associated with blunted cortisol reactivity to a laboratory stressor and resting cortisol levels in suicide attempters and ideators. Methods: 160 participants were recruited and grouped according to history of previous suicidal attempt, suicidal ideation or as control participants. Participants completed background questionnaires, including the Childhood Trauma Questionnaire, before completing a laboratory stress task. Cortisol levels were assessed at rest and during the stress task. Results: The highest levels of childhood trauma were reported in those who had attempted suicide (78.7\%), followed by those who thought about suicide (37.7\%) and then those with no suicidal history (17.8\%). Moreover, regression analyses showed that childhood trauma was a significant predictor of blunted cortisol reactivity to stress and resting cortisol levels, such that higher levels of trauma were associated with lower cortisol levels in those with a suicidal history. Family history of suicide did not interact with the effects of childhood trauma on cortisol levels. Conclusions: These results indicate that childhood trauma is associated with blunted HPA axis activity in vulnerable populations. The challenge for researchers is to elucidate the precise causal mechanisms linking trauma, cortisol and suicide risk and to investigate whether the effects of childhood trauma on cortisol levels are amendable to psychological intervention.
\end{abstract}

Keywords: chronic stress, adversity, HPA axis, suicide, depression, allostatic load 


\section{INTRODUCTION}

Suicide is a global health issue accounting for $1.5 \%$ of all mortality. Researchers have been investigating the causes of suicidal behavior for decades to identify risk factors in order to inform interventions. Recently, a small number of studies has explored whether cortisol reactivity to laboratory stressors may be associated with suicide attempt and ideation. For example, McGirr et al. (2010) investigated whether dysregulation of the HPA axis to a stressor was a heritable risk factor for suicidal behavior. In this study, a sample of first-degree relatives of those who died by suicide and matched controls were compared on their cortisol reactivity to an acute laboratory stressor. The results showed that the first-degree relatives exhibited a blunted cortisol response to the stressor. Another study of adult offspring of parents with mood disorder (Melhem et al., 2016) found the lowest levels of total cortisol output in the offspring who had attempted suicide (compared to the offspring with suiciderelated behavior but who had never attempted suicide, a non-suicidal offspring group and a healthy control group). However, they did not find a significant difference between the groups on a measure of cortisol reactivity to stress.

Most recently, O’Connor et al. (2017) investigated whether cortisol reactivity to a laboratory stressor differentiated individuals who had previously made a suicide attempt from those who had thought about suicide (suicide ideators) and control participants. The results showed that participants who had made a previous suicide attempt exhibited significantly lower cortisol levels in response to the stressor compared to participants in the control group. Suicide ideators were intermediate. Moreover, participants who made a suicide attempt and had a family history of suicide exhibited the lowest levels of cortisol in response to stress. In addition, Melhem et al. (2016) also found lower cortisol output in response to stress offspring of parents with mood disorder who also had a parent with a history of suicide attempt. Taken together, the evidence is converging to suggest that low (or blunted) cortisol responsiveness 
to stress is associated with aspects of suicide behaviour in adults and that aspects of the dysregulation of the stress response system may be a heritable risk factor for suicide risk.

An important next step for research in this area is to understand the factors that may contribute to HPA axis dysregulation in individuals vulnerable to suicide. A key candidate is likely to be childhood trauma. Numerous studies have linked childhood trauma to suicide risk, as well as to depression and psychopathology in adulthood (e.g., Carr et al., 2013; Heim et al., 2008; Marshall et al., 2013). For example, a large retrospective cohort study showed that adverse childhood experiences (e.g., abuse, neglect) increased risk of attempted suicide 2- to 5-fold throughout the lifespan (Dube et al., 2001). A systematic review found that childhood trauma, including sexual abuse, physical abuse, emotional abuse, physical neglect, and emotional neglect, triggers, maintains and increases the recurrence of psychiatric disorders (Carr et al., 2013). More recently, Marshall and colleagues (2013), in a prospective cohort study, found that severe sexual, physical and emotional childhood abuse conferred a substantial increased risk of suicide risk in illicit drug users.

Childhood trauma has been linked clearly to altered dynamics of the HPA axis and to persistent sensitization of the stress response system within the context of major depression (Heim et al., 2000; Heim et al., 2008). In addition, the effect of childhood trauma on depression has also been explained by changes in glucocorticoid resistance, increased central corticotropin-releasing factor (CRF) activity, immune activation, and reduced hippocampal volume. However, the results are mixed in the context of childhood trauma and cortisol reactivity. For example, Heim et al. (2000) showed that women who had a history of childhood abuse, with and without major depression, exhibited increased cortisol to an acute laboratory stressor (i.e., the Trier Social Stress Test; TSST). Whereas, a study by Carpenter et al. (2007) showed decreased cortisol levels in response to a laboratory stressor in childhood maltreated men who were never depressed. In a later study, Carpenter et al. (2011) replicated 
this finding and showed that women reporting childhood physical abuse displayed a blunted cortisol response to the TSST compared to women without physical abuse. More recently, other studies have begun to emerge suggesting that early life adversity is associated with blunted cortisol reactivity to stress (e.g., Lovallo et al., 2012; see also Lovallo, 2013 for a review). Data from the Oklahoma Family Health Patterns Project showed that experience of adversity predicted reduced cortisol response to laboratory stress challenge (Lovallo et al., 2012).

In addition to cortisol reactivity to stress, evidence is also converging to suggest that resting cortisol levels are also associated with suicide behavior (O'Connor et al., 2016). A study by Keilp et al. (2016) found evidence of low baseline cortisol levels in those who have attempted suicide compared to non-attempters (Keilp et al., 2016). Similarly, Lindqvist et al. (2008) showed that low cortisol activity was associated with suicidal behavior. More recently, Melhem et al. (2017) reported that those with a suicide attempt history had lower hair cortisol concentrations compared to controls (as well as ideators). However, similar to the cortisol reactivity studies in this area, less is known about the factors that may contribute to HPA axis dysregulation in individuals vulnerable to suicide.

Therefore, using data from the recent O'Connor et al. (2017) study, the primary aim of the current paper was to investigate whether childhood trauma was associated with low levels of cortisol reactivity to a laboratory stressor and lower resting cortisol levels in individuals vulnerable to suicide. Moreover, we were interested in exploring whether any observed relationships held while controlling for recent levels of depression and hopelessness. The secondary aim of the current study was to investigate whether family history of suicide interacted with childhood trauma in order to predict additional variability in levels of resting cortisol and cortisol reactivity to stress. 


\section{METHOD}

\subsection{Design and Participants}

Full details of the method have been reported elsewhere, therefore, only a brief summary is presented here (see O'Connor et al., 2017). One hundred and sixty participants (100 females) were recruited to a previous attempt $(n=49)$, a suicidal ideation but no attempt $(n=55)$ and a control group $(n=48)$ based upon established measures of suicidal behavior (see below $)$. Participants were aged between 18 and 62 years $(M=26.84$ years, $S D=9.32)$ with $73.8 \%$ identified as Caucasian. Participants were enrolled to the study in response to a local advertising campaign on websites (e.g., Gumtree, Twitter), via posters, flyers and emails. Eligible participants were required to be at least 18 years old and to understand English. Suicide ideation and attempt were assessed using the Self-Injurious Thoughts and Behaviors Interview (SITBI; Nock et al., 2007) and the Beck Scale for Suicide Ideation (Beck et al., 1979; Beck et al., 1988). Participants were allocated to the previous attempt group if they reported attempting to take their own life in the past or to the ideation group if they reported having thoughts of killing their self in the past 12 months. Participants were recruited to a control condition who reported no history of suicide attempt or ideation (and did not report any current psychiatric or psychological conditions). The current study was approved by the Research Ethics Committee of the School of Psychology, University of Leeds and the US Army Medical Research and Materiel Command, Office of Research Protections, Human Research Protection Office.

Of the 160 participants recruited to the study, 6 participants withdrew due to having a negative reaction to the Maastricht Acute Stress Test (e.g., felt faint, or did not want to take part in all of the stress test), 8 other participants were unable to be clearly allocated to any of the conditions (e.g., reporting an inconsistent suicide history or changing their suicide history between screening and commencing the study) and 1 participant who had extreme cortisol 
values (e.g., exhibiting values of $45.80 \mathrm{nmol} / \mathrm{L}$ and $52.42 \mathrm{nmol} / \mathrm{L}$ which remained outside the distribution after log transformation). Following removal of these participants, the statistical analysis was conducted on 145 participants (control group $=45$, ideator group $=53$, attempter group $=47$; see Table 1 for demographics and Table 2 for descriptive statistics for the main study variables). In the attempter group, 14 reported an attempt within the previous 12 months and 33 reported an historical attempt. In terms of family history of suicide, 25 participants reported they had a first degree relative who had attempted or completed suicide $($ control group $=3[6.7 \%]$, ideator group $=6[11.3 \%]$, attempter group $=16[34 \%])$. At baseline, $26.2 \%(n=38)$ of participants reported using prescribed medication (control group = $5[11.1 \%]$, ideator group $=16[30.2 \%]$, attempter group $=17[36.2 \%])$.

2.2 Child Trauma Questionnaire (CTQ; Bernstein et al., 2003).

A brief 28-item self-report inventory was used to assess for a history of abuse or neglect in childhood or adolescence. The CTQ has five subscales relating to types of maltreatment: emotional, physical and sexual abuse and emotional and physical neglect, with five items for each subscale $(1=$ never true, $5=$ very often true $)$. It also includes a 3 -item minimisation/denial scale for detecting false-negative trauma reports. Each subscale has a cut-off score to indicate a level of severity of childhood trauma ranging from none (or minimal), low (to moderate), moderate (to severe) and severe (to extreme). In the current study, we were interested in exposure to any type of childhood trauma, therefore, we created a total childhood trauma score. For each subscale, participants who reported scores in the moderate or severe range received a score of 1 to indicate exposure to that type of trauma. The cut-off scores for each the subscales for moderate or severe were: emotional abuse $>12$, physical abuse $>9$, sexual abuse $>7$, emotional neglect $>14$, and physical neglect $>9$. As a result, scores ranged on the CTQ ranged from 0 to 5. Internal and test-retest reliabilities, and content and construct validities, have been established with data from over 2,000 
respondents, including both clinical and non-clinical groups (Bernstein et al., 2003). The Cronbach's alpha in the current sample ranged from 0.73 to 0.95 .

2.3 Beck Depression Inventory-II (BDI-II; Beck, Steer, \& Brown, 1996). This BDI is a 21-item measure of depressive symtomatology which has been shown to yield reliable, internally consistent and valid scores in adult (Dozois, Dobson, \& Ahnberg, 1998) and adolescent populations (Osman, Barrios, Gutierrez, Williams, \& Bailey, 2007). The Cronbach's alpha in the current sample was 0.95 .

2.4 Beck Hopelessness Scale (BHS; Beck, Weissman, Lester, \& Trexler, 1974). The BHS is a well-established 20-item measure of pessimism for the future, which has been shown to be predictive of repetitive self-harm and suicide. It has also been shown to have internal consistency and convergent validity (Steed, 2001). The Cronbach's alpha in the current sample was 0.88 .

\subsection{Maastricht Acute Stress Test (MAST, Smeets et al., 2012)}

The MAST is a recently developed stress protocol designed to be both physiologically and psychologically challenging by combining an uncontrollable physical stressor (i.e., a cold pressor challenge) with a social-evaluative (i.e., mental arithmetic) component (Smeets et al., 2012). In addition, it has been shown to yield similar subjective and cortisol stress responses to the Trier Social Stress Test, however, it does not require the presence of a panel (see Kirschbaum et al., 1993). The MAST is described further in the procedure section.

\subsection{Procedure}

After each participant provided their consent they were led into a quiet room that served as a rest area. Here the participant rested for 10 minutes to allow for any HPA axis activation to return to baseline levels. After 10 minutes the participant was asked to provide their first saliva sample (resting cortisol sample). Next the MAST was introduced to the 
participants. After a short preparation and anticipation phase (5 min), participants were asked to complete five socially evaluated cold pressor trials where participants immersed their hands in cold water for varying durations (60 to 90s) over a 10-minute time span. In between trials, participants were instructed to perform a mental arithmetic task as fast and as accurately as possible and received negative feedback on their performance when mistakes were made. To increase unpredictability and uncontrollability participants were told the duration and the order of the hand immersion trials and mental arithmetic task were randomly chosen by the computer, whereas in reality the order and duration of trials was fixed for all participants. To measure cortisol reactivity to stress, saliva samples were taken using Salivettes (Sarstedt, Germany) during the resting cortisol phase and then at the beginning of the stress task (T00), at $+10,+20,+30$ and +40 minutes post-task.

\subsection{Cortisol assay methods}

Cortisol samples were collected from saliva using Salivettes and were stored at $-20^{\circ} \mathrm{C}$ until assay. Cortisol levels were determined by using a competitive enzyme-linked immunosorbent assay kit (ELISA) designed for analysing saliva. Intra-assay and inter-assay coefficients of variation of this assay were $4.26 \%$ and $4.91 \%$, respectively. Note that all laboratory visits were scheduled after $11 \mathrm{am}$ in the morning (with $97 \%$ taking place between $11 \mathrm{am}$ and $3 \mathrm{pm}$ ) in order to ensure the sampling was not influenced by the cortisol awakening response.

\subsection{Statistical analysis}

Descriptive statistics were calculated for all study variables. The main analyses focused on the combined group of suicide attempters and ideators given that only 8 participants in the control condition reported exposure to any type of moderate or severe 
childhood trauma. Before conducting the statistical analyses the cortisol levels were log transformed due to skewness, however, non-transformed values are presented in Table 1 for ease of interpretation. In addition to the resting cortisol level, two area under the curve measures (AUC) were calculated. Using the five sampling time points, the two AUC measures were determined following established procedures (Gartland et al., 2014; O’Connor et al., 2013; Pruessner et al., 2003). Area under the curve with respect to ground (AUCg) is a measure of total cortisol output throughout the stressor and area under the curve with respect to increase (AUCi) captures the sensitivity of the HPA system and is a measure of its ability to change in response to the MAST.

Hierarchical linear regression was utilised, following the procedures outlined by Kenny et al. (1998), to test whether childhood trauma was associated with lower levels of cortisol reactivity to a laboratory stressor (AUCg and $\mathrm{AUCi}$ ) and lower resting cortisol levels in suicide attempters and ideators. First, in order to control for age, gender, BMI, medication usage, time of day and smoking status, these variables were entered in step 1 of the equation. Second, childhood trauma score was entered into the equation in step 2. Finally, to test whether any observed relationships held while controlling for recent levels of depression and hopelessness, these two variables were entered into the third and final step of each equation. In order to test the interactive effects of family history and childhood trauma on cortisol levels, the regression analyses were repeated with the same variables entered in step 1 and step 2, but with family history entered at step 3, and the multiplicative interaction family history x childhood trauma interaction entered at step 4.

[Insert Tables 1 and 2 about here ]

\section{RESULTS}

Descriptive statistics for the main study variables are presented in Table 2. 
In terms of childhood trauma scores, individuals in the suicide attempter group scored significantly higher on all subscales of the CTQ measure compared to individuals in the ideator and control groups, $\mathrm{F}(10,276)=6.07, \mathrm{p}<0.001$ (see Figure 1). Moreover, the ideator group was intermediate to the two other groups on all the subscales, however, their scores were only significantly different from controls for physical neglect $(\mathrm{p}<0.001)$, emotional abuse ( $\mathrm{p}=0.038$ ), and marginally for emotional neglect ( $\mathrm{p}=0.058)$. In terms of exposure to any type of childhood trauma (that was moderate or severe), the highest levels of trauma were reported in the attempter group $(78.7 \%)$, followed by the ideator $(37.7 \%)$ and then the control (17.8\%) groups.

\section{[ insert Figure $1 \& 2$ about here ]}

\subsection{Effects of childhood trauma on resting cortisol levels in combined group of suicide attempters and ideators}

As outlined earlier, the effects of childhood trauma on cortisol outcomes were examined using hierarchical regression. As shown in Table 3, at step 1, the control variables together did not significantly enter the equation, although, they accounted for $9 \%$ of the variance, $F(6,93)=1.58, p=0.16$. At step 2 , as predicted, childhood trauma $(\beta=-0.24, \mathrm{p}=$ 0.02 ) significantly entered the equation, $F(1,92)=5.47, p=0.02$, and explained an additional $5 \%$ in the variability in resting cortisol levels, indicating higher levels of trauma were associated with lower cortisol levels. In the final step, depression and hopelessness did not significantly enter the equation, $F(2,90)=2.05, p=0.13$, although, the effect of hopelessness was statistically significant $(\beta=-0.26, \mathrm{p}=0.047)$. However, importantly, the effects of childhood trauma remained significant when depression and hopelessness were accounted for in the final equation.

[ Insert Tables 3 and 4 about here ] 
3.2 Effects of childhood trauma on cortisol reactivity to stress in combined group of suicide attempters and ideators

For AUCg, at step 1 , gender $(\beta=-0.24, p=0.02)$ and medication usage $(\beta=0.24, p=$ 0.02) significantly entered the regression equation, and together with the other control variables accounted for $13 \%$ of the variance, $F(6,93)=2.28, p=0.04$ (see Table 4 ). At step 2 , again as predicted, childhood trauma $(\beta=-0.23, \mathrm{p}=0.02)$ significantly entered the equation, $F(1,92)=5.32, p=0.02$, and explained an additional $5 \%$ in the variability in AUCg levels, indicating higher levels of trauma were associated with lower cortisol levels. In the final step, depression and hopelessness did not significantly enter the equation, $F(2,90)$ $=0.93, p=0.40$ (see Figure 3 for a graphical representation based upon different levels of childhood trauma exposure). However, again, the effects of childhood trauma remained significant when depression and hopelessness were accounted for in the final equation.

For AUCi, at step 1, none of the control variables significantly entered the regression equation, $F(6,93)=1.08, p=0.38$. At step 2 , childhood trauma did not significantly enter the equation, $F(1,92)=0.25, p=0.62$. Finally, in step 3, depression and hopelessness did not significantly enter the equation, $F(2,90)=0.91, p=0.41$.

\subsection{Effects of family history of suicide and childhood trauma on resting cortisol and cortisol reactivity to stress in combined group of suicide ideators and attempters}

As outlined above, the regression analyses were repeated but with family history entered at step 3, and the family history $\mathrm{x}$ childhood trauma interaction entered at step 4. For resting cortisol levels, at step 3 , family history $(\beta=0.07, p=0.48)$ did not significantly enter the equation, $F(1,91)=0.50, p=0.48$. Similarly, at step 4 , the family history $\mathrm{x}$ childhood trauma interaction $(\beta=0.01, \mathrm{p}=0.94)$ did not significantly enter the equation, $F(1,90)=$ $0.01, p=0.94$. For AUCg, at step 3, family history $(\beta=0.07, \mathrm{p}=0.48)$ did not significantly 
enter the equation, $F(1,91)=0.50, p=0.48$. Similarly, at step 4 , the family history $\mathrm{x}$ childhood trauma interaction $(\beta=0.01, \mathrm{p}=0.94)$ did not significantly enter the equation, $F$ $(1,90)=-0.01, p=0.94$. Finally, for AUCi, at step 3 , family history $(\beta=-0.19, p=0.07)$ did not significantly enter the equation, $F(1,91)=3.40, p=0.07$ and, at step 4 , the family history $\mathrm{x}$ childhood trauma interaction $(\beta=-0.29, \mathrm{p}=0.10)$ did not significantly enter the equation, $F(1,90)=2.85, p=0.10$.

\section{DISCUSSION}

Three main findings emerged from the current study. First, high levels of childhood trauma were reported in individuals vulnerable to suicide, in particular, in individuals who had previously made a suicide attempt. Second, higher levels of childhood trauma were found to be associated with lower resting cortisol and blunted cortisol reactivity to stress (while controlling for age, gender, BMI, medication usage, time of day and smoking status). Third, family history of suicide did not interact with childhood trauma to predict additional variability in (low) levels of cortisol at rest or in response to stress.

The highest levels of childhood trauma were reported in the group who had attempted suicide. Specifically, $78.7 \%$ of participants in the suicide attempt group reported exposure to at least one type of childhood trauma that was classified as moderate or severe compared to $37.7 \%$ and $17.8 \%$ in the ideator and control groups, respectively. Such high levels of childhood trauma in suicide attempters are consistent with existing published studies (e.g., Enns et al., 2006; Hassan, Stuart \& De Luca, 2016; Marshall et al., 2013; Sachiapone et al., 2007). For example, Marshall et al (2013), using the CTQ, found similarly high levels of moderate and severe childhood trauma being associated with suicide attempt in a prospective cohort study of illicit drug users. In particular, they showed that severe sexual, physical and emotional childhood abuse conferred a substantial increased repeated suicide risk in 
adulthood. An earlier study by Sachiapone et al. (2007) also found that higher scores on the CTQ were associated with suicide attempt in patients with unipolar depression. Moreover, high levels of emotional abuse and emotional neglect were identified as being particularly important.

Similar results, using a different measure of childhood trauma, were also found in a large longitudinal population-based study in the Netherlands (Enns et al., 2006). In this study childhood neglect, psychological abuse and physical abuse were shown to be strongly associated with new onset suicide ideation and suicide attempt over a 3 year follow-up. In fact, these authors concluded that the total number of childhood adversities (in particular, childhood abuse/trauma) had a strong graded relationship with suicide attempts (as well as with suicide ideation). Therefore, taken together, these studies suggest that childhood trauma may have an additive, cumulative effect on suicide risk, whereby exposure to a greater number of childhood adversities may increase vulnerability to suicide. However, much remains to be learned about factors that mediate the association between childhood trauma and increased vulnerability to suicide. For example, what are the precise mechanisms that confer this increased risk? Do individuals who experience serious childhood adversities become sensitized to the negative effects of acute stress during late adolescence and early adulthood?

The main finding from the current study was that higher levels of childhood trauma were associated with lower resting cortisol and blunted cortisol reactivity to stress (while controlling for age, gender, BMI, time of day, medication \& smoking status). In particular, individuals who reported more than one moderate or severe type of childhood trauma exhibited the lowest cortisol levels in response to stress and at rest (see Figure 3). Notably the observed effects were not explained by current levels of depression or hopelessness. Our findings are consistent with the work of Carpenter et al. (2007) who showed that childhood 
trauma, assessed using the CTQ, was associated with lower secretion of cortisol (and adrenocorticotropin hormone) in response to a laboratory stress challenge (i.e., TSST). More recently, the same group published another study in a different sample, demonstrating that physical abuse in childhood, in particular, was also associated with a blunted cortisol response to the TSST (Carpenter et al., 2011). Further evidence of strong links between early life adversity and reduced cortisol reactivity to stress comes from the Oklahoma Family Health Patterns Project which also showed that experience of adversity predicted reduced cortisol response to laboratory stress challenge (Lovallo et al., 2012).

This pattern of results is consistent with an important earlier finding by McGowan and colleagues (2009) in an investigation of postmortem hippocampus in individuals who died by suicide with and without a history of childhood abuse. Specifically, these authors showed that hippocampal glucocorticoid receptor expression was lowest in individuals who died by suicide and had a history of childhood abuse. Moreover, no differences in glucocorticoid receptor expression were observed between individuals who died by suicide without a history of childhood abuse and controls. These findings suggest that exposure to early life adversity may be more closely related to glucocorticoid receptor expression, and as a result HPA axis activity, than completed suicide.

In contrast, a number of studies published by Heim et al. have shown that childhood trauma, in depressed and never-depressed women, have been associated with hyperactivity of the HPA axis in response to stress (for a review, see Heim et al., 2008). In addition, studies in post-traumatic stress disorder patients have also found cortisol hyperactivity following a stress challenge (e.g., de Kloet et al., 2006). It is difficult to reconcile our current findings, the work by Carpenter, Lovallo and colleagues with that of Heim and colleagues. One possibility is that these discrepancies are accounted for by differences in the samples under 
investigation. Many of the studies described by Heim et al. (2008) have focussed on patients with clinical depression and these authors have argued that stratifying groups based upon childhood trauma and major depression is important. For example, in their earlier work Heim showed that changes in stress response systems (i.e., hyperactivity) were only observed in depressed patients with childhood trauma, but not in depressed patients without trauma. The elevated stress responses in patients with major depression and trauma are likely to be explained by glucocorticoid receptor dysfunction, frequently observed in patients with major depression, that leads to disrupted glucocorticoid-mediated negative feedback (Heim et al., 2008). If this is the case, it is possible that different mechanisms are at play in individuals with childhood trauma depending on whether they are clinically depressed or not. Heim and colleagues have suggested that sex differences, variations in type or timing of trauma(s) and/or genetic factors might also contribute to these divergent findings. Indeed, similar arguments have been proffered in the context of inconsistent findings in the cortisol and suicide attempt literature (e.g., O’Connor et al., 2016).

We believe the current findings are important as they suggest that the experience of childhood trauma may predispose individuals to vulnerability to suicide by leading to blunted HPA axis activity in response to stress and during rest. Evidence is accumulating that repeated activation of the HPA axis leads to dysregulation (e.g., Hwang et al., 2014; McEwen, 1998; 2000; Miller et al., 2007). This is best articulated in the context of McEwen's concept of allostatic load, whereby if the HPA axis is repeatedly activated (by chronic stress) the immune, cardiovascular and the endocrine systems are potentially exposed to excessive demands that over time can lead to dysregulation of these systems (McEwen, 1998; 2000). In terms of cortisol reactivity to stress, there has been much debate about whether dysregulation related to exposure to chronic stress leads to hypercortisolism (enhanced secretion) or hypocortisolism (blunted secretion; Miller et al., 2007; O’Connor et al., 2009; 2016; 
Segerstrom and Miller, 2004). The evidence is now converging to suggest that repetitive and sustained activation of the HPA axis stress system causes a blunted or reduced cortisol response over time (Fries et al., 2005; Hwang et al., 2014; O’Connor et al., 2009; 2013). Indeed, this view is also in line with an influential account of the development of hypocortisolism, which suggests that the latter phenomenon occurs after a prolonged period of hyperactivity of the HPA axis due to chronic stress (see Fries et al., 2005, for detailed discussion of precise mechanisms). Therefore, in the current context, it is possible that in individuals who have experienced greater levels of childhood trauma, over a more sustained period, their HPA axis may have become dysregulated leading to lower levels of cortisol secretion at rest and in response to stress.

An important next step for research in this area is to elucidate the precise causal mechanisms linking childhood trauma, low levels of cortisol and suicide risk. A number of candidate mechanisms have been tentatively suggested (e.g., impaired executive function, increased impulsiveness). For example, McGirr et al. (2010) showed that a sample of firstdegree relatives of those who died by suicide exhibited a blunted cortisol response to stress and they also displayed evidence of impairment in aspects of executive function (i.e., they did not improve on measures of inhibition upon repeated testing after the TSST). Relatedly, Lovallo and colleagues $(2012 ; 2013)$ showed that exposure to early adversity was associated with blunted cortisol reactivity to stress and altered cognitive function in the form of poorer working memory, greater impulsive behaviours and lower general intelligence. In the same sample, these authors also found evidence linking exposure to adversity to more disinhibited lifestyles, less stable mood regulation and being less socially connected - all variables linked to suicide behaviour (Lovallo et al., 2013). More specifically, Lovallo (2013), from the perspective of addiction research, has suggested a conceptual model that summarises how adverse life experiences in childhood and adolescence can modify behaviour patterns that 
ultimately lead to adverse health outcomes. Put simply, he argues that adverse life experiences cause modifications in frontolimbic brain function which may then lead directly to: 1) reduced stress reactivity, 2) altered cognition (characterised by a shift in focus to more short term goals and impulsive response selection) and 3) unstable affect regulation. Taken together, these three negative consequences contribute to the development of a more impulsive behavioural style that may increase risk of addiction and the engagement in poor health behaviours. We contend that the development of a more impulsive behavioural style, and each of the precursors outlined above, are likely to also increase risk of suicide behavior. Therefore, the current findings relating to blunted HPA axis activity in response to stress and during rest in individuals with high levels of childhood trauma provide further evidence for one of the pathways suggested by Lovallo (2013) and extend his model to suicide behavior. Future research ought to utilise Lovallo's framework and examine further how precisely childhood trauma-induced cortisol dysregulation leads to increased suicide risk. Moreover, the current findings are also consistent with a recently published wide-ranging review paper that comprehensively summarises recent work that clearly demonstrates that blunted cortisol reactions to acute stress (as well as blunted cardiovascular reactions) are associated with a range of adverse behavioural and health outcomes including addiction, depression, neuroticism and impulsivity (Carroll et al., 2017). These authors build upon and expand on the theorising of Lovallo (2013) and provide compelling evidence to suggest that blunted stress reactivity is a possible marker for more central dysfunction. It is also notable that in their concluding remarks and future directions the authors comment "Although preliminary evidence is emerging, there needs to be much more focus on the association between blunted stress responding and early life adversity, particularly emotional neglect and abuse" (Carroll et al., 2017; p. 82). 
Another important next step for research in this area is to investigate whether HPA axis dysregulation is amendable to psychological and/or pharmacological intervention. We have previously shown, in the same sample, that cortisol levels following stress challenge are lower (and blunted) in individuals who have made a recent suicide attempt (i.e., within the past year) compared to those with an historical attempt (O'Connor et al., 2017). Indeed cortisol levels in those who have made an historical attempt appeared to be closer to those who have no history of suicide behaviour. This suggests that psychological and/or pharmacological intervention may yield benefits over time and help facilitate (partial) recovery of the HPA axis stress response system. Therefore, we would urge researchers to investigate the effectiveness of psychological interventions, particularly therapies aimed at treating childhood trauma, and utilise a combination of psychological and endocrine assessments in order to fully understand the pathways linking early adversity to suicide behaviour.

We also found that family history did not interact with childhood trauma to explain any additional variability in cortisol levels in response to stress or at rest. Previously in this sample, we have reported that having a family history of suicide was associated with making a suicide attempt and with exhibiting the lowest cortisol response to stress in the laboratory. This finding was in line with another study by McGirr et al. (2010) who found that first degree relatives of suicide completers also showed a blunted cortisol (and $\alpha$ amylase) response to an acute laboratory stressor. Similarly, Melhem et al. (2016) also found lower cortisol output in response to stress in offspring of parents with mood disorder who also had a parent with a history of suicide attempt. Overall, these findings suggest that dysregulation of the HPA axis may be, in part, heritable and confer additional vulnerability to suicide behaviour. Therefore, we were interested in exploring whether individuals who had a family history of suicide and were exposed to moderate or severe childhood trauma exhibited a more 
blunted response to stress compared to participants who had no family history. As indicated above however, we found little or no evidence of an interaction suggesting that the effects of childhood trauma and family history on HPA axis dysregulation are independent of each other. Nevertheless, we accept that one of the interactions was marginal (AUCi, $p=0.07$ ) and these analyses may have been underpowered due to the relatively small sample size. Therefore, we would suggest that future researchers investigate this proposed interaction in larger samples of individuals who are vulnerable to suicide before drawing any firm conclusions about the role of family history of suicide in this context.

We acknowledge that there are a number of limitations of the current study that require further comment. We recognise that we were unable to investigate the independent effects of the different types of childhood trauma given that our main analyses were focused only on 100 vulnerable participants. However, our approach was similar to other researchers who have focused on the total number of childhood adversities experienced (e.g., Enns et al., 2006; Lovallo et al., 2012; Sachiapone et al., 2007). Moreover, in terms of experimental research in the suicide area, this represents a relatively large sample (cf., O'Connor et al., 2016). We are also cognisant of the fact that the CTQ is a retrospective self-report tool that is open to demand characteristics, social (un)desirability, repression and memory biases, and as such, we cannot confirm the veracity of the reports or if they are influenced by current levels of distress. However, reassuringly, it is important to note that the observed effects of childhood trauma on cortisol levels remained statistically significant when depression and hopelessness were controlled and, if anything, retrospective self-report tools may lead to an underestimation of actual occurrence (Hardt \& Rutter, 2004). We also recognise that the resting baseline period in the current study design could have been longer to increase the likelihood that the participants had reached a fully rested state. In addition, we acknowledge that the resting baseline sample may have been elevated, relative to a sample taken on a 
control day, given participants were exposed to a novel experimental environment on the same day the resting sample was taken. However, it is worth noting that it is unlikely that these issues would have differentially impacted on our results in relation to suicide attempt status and exposure to childhood trauma.

In conclusion, this study showed that very high levels of childhood trauma were observed in individuals vulnerable to suicide, in particular, in individuals who had previously made a suicide attempt. Higher levels of childhood trauma were associated with blunted HPA axis activity in vulnerable populations and these effects were independent of family history of suicide. The challenge for researchers is to elucidate the precise causal mechanisms linking trauma, cortisol and suicide risk and to investigate whether the effects of childhood trauma on cortisol levels are amendable to psychological intervention. 


\section{Authors' contributions}

All authors contributed equally

\section{Acknowledgements}

This research was supported in part by a research award from the US Department of Defense (Award No. W81XWH-12-1-0007). Opinions, interpretations, conclusions and recommendations are those of the authors and are not necessarily endorsed by the funder. The funder had no role in the writing of the manuscript.

\section{Conflict of interest}

None

\section{Ethical standards}

The authors assert that all procedures contributing to this work comply with the ethical standards of the relevant national and institutional committees on human experimentation and with the Helsinki Declaration of 1975, as revised in 2008. 


\section{REFERENCES}

Beck, A.T., Kovacs, M., Weissman, A. 1979. Assessment of suicidal intention: The scale of suicide ideation. J Consult Clin Psychol, 47, 343-352.

Beck, A.T., Steer, R.A., Rantieri, W.F. 1988. Scale for suicide ideation: Psychometric properties of a self-report version. J Clin Psychol, 44, 499-505.

Beck, A. T., Steer, R. A., Brown, G. K. 1996. Manual for the Beck Depression Inventory-II. San Antonio, TX: Psychological Corporation.

Beck, A.T., Weissman, A., Lester, D., Trexler, L. 1974. The measurement of pessimism: The Hopelessness Scale. J Consult Clin Psychol, 42, 861-865.

Bernstein, D. P., Stein, J. A., Newcomb, M. D., Walker, E., Pogge, D., Ahluvalia, T., Stokes, J., Handelsman, L., Medrano, M., Desmond, D., Zule, W. 2003. Development and validation of a brief screening version of the Childhood Trauma Questionnaire. Child Abuse \& Neglect, 27, 169-190.

Carr, C.P., Martins, C.M., Stingel, A.M., Lemgruber, V.B., Juruena, M.F. 2013. The role of early life stress in adult psychiatric disorders: A systematic review according to childhood trauma subtypes. J Nerv Mental Disord, 201, 1007-1020.

Carroll, D., Ginty, A.T., Whittaker, A.C., Lovallo, W.R., de Rooji, S. (2017). The behavioural, cognitive, and neural corollaries of blunted cardiovascular and cortisol reactions to acute psychological stress. Neurosci Biobehav Rev, 77, 74-86.

Carpenter, L.L., Shattuck, T.T., Tyrka, A.R., Geracioti, T.D., Price, L.H. 2011. Effect of childhood physical abuse on cortisol stress response. Psychopharmacology, 214, 367-375.

Carpenter, L.L., Carvalho, J.P., Tyrka, A.R., Wier, L.M., Mello, A.F., Mello, M.F., Anderson, G.M., Wilkinson, C.W., Price, L.H. 2007. Decreased adrenocorticotropic hormone and cortisol responses to stress in healthy adults reporting significant childhood maltreatment. Biol Psychiatry, 62, 1080-1087.

de Kloet, C.S., Vermetten, E., Geuze, E., Kavelaars, A., Heijnen, C.J., Westenberg, H.G. 2006. Assessment of HPA-axis function in posttraumatic stress disorder: Pharmacological and non-pharmacological challenge tests, a review. J Psychiatry Res, 40, $550-567$.

Dozois, D. J. A., Dobson, K. S., Ahnberg, J. L. 1998. A psychometric evaluation of the Beck Depression Inventory-II. Psychol Assess, 10, 83-89.

Dube, S.R., Anda, R.F., Felitti, V.J., Chapman, D.P., Williamson, D.F., Giles, W.H. 2001. Childhood abuse, household dysfunction, and the risk of attempted suicide throughout the life span: findings from the Adverse Childhood Experiences Study. JAMA, 286, 3089-3096. 
Enns, M.W., Cox, B.J., Afifi, T.O., de Graff, R., ten Have, M. \& Sareen, J. 2006. Childhood adversities and risk for suicide ideation and attempts: a longitudinal population-based study. Psychol Med, 36, 1769-1778.

Fries, E., Hesse, J., Hellhammer, J., Hellhammer, D.H. 2005. A new view of hypocortisolism. Psychoneuroendocrinology, 30, 1010-1016.

Gartland, N., O’Connor, D.B., Lawton, R., Bristow, M. 2014. Exploring day-to-day dynamics of daily stressor appraisals, physical symptoms and the cortisol awakening response.

Psychoneuroendocrinology, 50, 130-138.

Hardt, J., Rutter, M. 2004. Validity of adult retrospective reports of adverse childhood experiences: review of the evidence. J Child Psychol Psychiatry, 45, 260-273.

Hassan, A.N., Stuart, E.A., \& De Luca, V. 2016. Childhood maltreatment increases the risk of suicide attempt in schizophrenia. Schizophrenia Res, 176, 572-577.

Heim, C., Newport, D.J., Heit, S., Graham, Y.P., Wilcox, M., Bonsall, R., Miller, A.H., \& Nemeroff, C.B. 2000. Pituitary-adrenal and autonomic responses to stress in women after sexual and physical abuse in childhood. JAMA, 284, 592-597.

Heim, C., Newport, D.J., Mletzko, T., Miller, A.H., \& Nemeroff, C.B. 2008. The link between childhood trauma and depression: Insights from HPA axis studies in humans. Psychoneuroendocrinology, 33, 693-710.

Hwang, A., Peng, L., Wen, Y., Tsai, Y., Chang, L., Chiou, S., Chen, L. 2014. Predicting allcause and cause specific mortality by static and dynamic measurements of allostatic load: A 10-year population-based cohort study in Taiwan. J Am Med Dir Assoc, 15, 490-496.

Keilp, J.G., Stanley, B.H., Beers, S.R., Melhem, N.H., Burke, A.K., Cooper, T.B., Oquendo, M.A., Brent, D.A., Mann, J.J. 2016. Further evidence of low baseline cortisol levels in suicide attempters. J Affect Disord, 190, 187-192.

Kenny, D. A., Kashy, D. A., \& Bolger, N. 1998. Data analysis in social psychology. In D. T. Gilbert, S. T. Fiske, \& G. Lindzey (Eds.), The handbook of social psychology (pp. 233-265). Boston, MA: McGraw-Hill.

Kirschbaum, C., Pirke, K.M., Hellhammer, D.H. 1993. The 'Trier Social Stress Test' —a tool for investigating psychobiological stress responses in a laboratory setting.

Neuropsychobiology 28, 76-81.

Lindqvist, D., Isaksson, A., Träskman-Bendz, L., Brundin, L. 2008. Salivary cortisol and suicidal behavior--a follow-up study. Psychoneuroendocrinology, 33, 1061-8.

Lovallo, W.R., Farag, N.H., Sorocco, K.H., Cohoon, A.J., Vincent, A.S. 2012. Lifetime adversity leads to blunted stress axis reactivity: Studies from the Oklahoma Family Health Patterns Project. Biol Psychiatry, 71, 344-349.

Lovallo, W.R., Farag, N.H., Sorocco, K.H., Acheson, A., Cohoon, A.J., Vincent, A.S. 2013. Early life adversity contributes to impaired cognition and impulsive behavior in persons with 
a family history of alcoholism: Studies from the Oklahoma Family Health Patterns Project. Alcohol Clin Exper Res, 37, 616-623.

Lovallo, W.R. 2013. Early life adversity reduces stress reactivity and enhances impulsive behaivor: Implications for health behaviors. Int J Psychophysiology, 90, 8-16.

Mann, J.J., Waternaux, C., Haas, G.L., Malone, K.M. 1999. Toward a clinical model of suicidal behavior in psychiatric patients. Am J Psychiatry, 156, 181-189.

Marshall, B.D.L., Galea, S., Wood, E., Kerr, T. 2013. Longitudinal associations between types of childhood trauma and suicidal behaviour among substance users: A cohort study. Am J Pub Health, 103, e69-e75.

McEwen, B.S. 1998. Protective and damaging effects of stress mediators. NEJM, 338, 171179.

McEwen, B.S. 2000. Allostasis and allostatic load: Implications for neuropsychopharmacology. Neuropsychopharmacology 22, 108-124.

McGirr, A., Diaconu, G., Berlim, M.T., Pruessner, J.C., Sable, R., Cabot, S., Turecki, G. 2010. Dysregulation of the sympathetic nervous system, hypothalamic-pituitary-adrenal axis and executive function in individuals at risk for suicide. J Psychiatry Neurosci, 35, 399-408.

McGowan, PO., Sasaki, A., D'Alessio, A.C, Dymov, A.C., Labonte, B., Szyf, M., Turecki, G., Meaney, M.J. (2009). Epigenetic regulation of the glucocorticoid receptor in human brain associates with childhood abuse. Nature Neurosci,12, 342-348.

Melhem, N.M., Keilp, J.G., Porta, G., Oquendo, M.A., Burke, A., Stanley, B., Cooper, T.B., Mann, J.J., Brent, D.A. 2016. Blunted HPA Axis Activity in Suicide Attempters Compared to those at High Risk for Suicidal Behavior. Neuropsychopharmacology, 41, 1447-56.

Melham, N.M., Munroe, S., Marsland, A., Gray, K., Brent, D., Porta, G., Douaihy, A., Laudenslager, M.L., DePietro, F., Diler, R., Driscoll, H., Gopalan, P. 2017. Blunted HPA axis activity prior to suicide attempt and increased inflammation in attempters. Psychoneuroendocrinology, 77, 284-294.

Miller, G.E., Chen, E., Zhou, E.S. 2007. If it goes up, must it come down? Chronic stress and the hypothalamic-pituitary-adrenocortical axis in humans. Psych Bull, 133, 25-45.

Nock, M.K., Holmberg, E.B., Photos, V.I., Michel, B.D. 2007. Self-injurious thoughts and behavior interview: development, reliability, and validity in an adolescent sample. Psychol Assess, 19, 309-317.

Pruessner, J.C., Kirschbaum, C., Meinlschmid, G., Hellhammer,D.H. 2003. Two formulas for computation of the area underthe curve represent measures of total hormone concentration versus time-dependent change. Psychoneuroendocrinology, 28, 916-931.

O’Connor, D.B., Hendrickx, H., Dadd, T., Talbot, D., Mayes, A., Elliman, T., Willis, T., Dye, L. 2009. Cortisol awakening rise in middle-aged women in relation to chronic psychological stress. Psychoneuroendocrinology 34, 1486-1494. 
O’Connor, D.B., Ferguson, E., Green, J., O’Carroll, R.E., O’Connor, R.C. 2016. Cortisol and suicidal behavior: A meta-analysis. Psychoneuroendocrinology, 63, 370-379.

O’Connor, D.B., Green, J., Ferguson, E., O’Carroll, R.E., O’Connor, R.C. 2017. Cortisol reactivity and suicidal behavior: investigating the role of hypothalamic-pituitary-adrenal (HPA) axis responses to stress in suicide attempters and ideators, Psychoneuroendocrinology, $75,183-191$.

O’Connor, D.B., Walker, S., Hendrickx, H., Talbot, D., Schaefer, A. 2013. Stress-related thinking predicts the cortisol awakening response and somatic symptoms in healthy adults. Psychoneuroendocrinology, 38, 438-446.

O'Connor, R.C. 2011. Towards an Integrated Motivational-Volitional of Suicidal Behaviour. In R O'Connor, S Platt, \& J Gordon (Eds.) International Handbook of Suicide Prevention: Research, Policy and Practice. Wiley Blackwell. pp181-198.

O’Connor, R.C., Nock, M. 2014. The psychology of suicidal behaviour. Lancet Psychiatry, 1, 73-85.

Osman, A., Barrios, F. X., Gutierrez, P. M., Williams, J. E., Bailey, J. 2007. Psychometric properties of the Beck Depression Inventory-II in nonclinical adolescent samples. J Clin Psychol, 64, 83-102.

Sachiapone, M., Carli, V., Cuomo, C., Roy, A. 2007. Childhood trauma and suicide attempts in patients with unipolar depression. Depress Anxiety, 24, 268-272.

Saraçlı, Ö., Atasoy, N., Şenormancı, Öm., Atik, L., Açıkgöz, H. O., Doğan, V., Sankır, H., Köktürk, F., Örsel, S. 2016 Childhood trauma and suicide risk in the population living in Zonguldak Province. Asia-Pacific Psychiatry, 8, 136-144.

Segerstrom, S., Miller, G.E. 2004. Psychological Stress and the Human Immune System: A Meta-Analytic Study of 30 Years of Inquiry. Psychol Bull, 130, 601-630.

Smeets, T., Cornelisse, S., Quaedflieg, C., Meyer, T., Jelicic, M., Merckelbach, H. 2012. Introducing the Maastricht Acute Stress Test (MAST): A quick and non-invasive approach to elicit robust autonomic and glucocorticoid stress responses. Psychoneuroendocrinology 37, 1998-2008.

Steed, L. 2001. Further validity and reliability evidence for Beck Hopelessness Scale scores in a nonclinical sample. Educ Psychol Measurement, 61, 303-316.

van Heeringen, K., Mann, J.J. 2014. The neurobiology of suicide. Lancet Psychiatry, 1, 6372.

van Orden, K.A., Witte, T.K., Cukrowicz, K.C., Braithwaite, S.R., Selby, E.A., \& Joiner, T.E. Jr. 2010. The interpersonal theory of suicide. Psychol Rev, 117, 575-600.

World Health Organization 2014. Preventing suicide: a global imperative. Geneva, Switzerland. 
Table 1. Baseline characteristics for participants in each study group $(n=145)$

\begin{tabular}{|c|c|c|c|}
\hline Characteristic & $\begin{array}{c}\text { Control } \\
\text { group } \\
(\mathrm{n}=45) \\
\end{array}$ & $\begin{array}{c}\text { Ideator } \\
\text { group } \\
(\mathrm{n}=53)\end{array}$ & $\begin{array}{l}\text { Attempter } \\
\text { group } \\
(\mathrm{n}=47)\end{array}$ \\
\hline Age (SD) & $24(8)$ & $28(8)$ & $28(10)$ \\
\hline Sex $(\%$ female $)$ & $29(64.4)$ & $29(54.7)$ & $30(63.8)$ \\
\hline \multicolumn{4}{|l|}{$\begin{array}{l}\text { Current psychiatric/psychological } \\
\text { diagnosis* }\end{array}$} \\
\hline Depression & 0 & 16 & 8 \\
\hline Anxiety & 0 & 9 & 3 \\
\hline Bipolar disorder & 0 & 0 & 4 \\
\hline Personality disorder & 0 & 0 & 3 \\
\hline $\begin{array}{l}\text { Number of lifetime attempts } \\
\\
\\
\text { Method in most recent attempt } \\
\text { Own prescription drugs } \\
\text { Over-counter drugs } \\
\text { Poison } \\
\text { Immolation } \\
\text { Hanging } \\
\text { Sharp object } \\
\text { Auto exhaust } \\
\text { Suffocation } \\
\text { Alcohol } \\
\text { Salt water } \\
\text { Multiple methods }\end{array}$ & & & $\begin{array}{c}1 \text { attempt }=21 \\
2 \text { attempts }=6 \\
3 \text { attempts }=5 \\
4 \text { attempts }=2 \\
\geq 5 \text { attempts }=13 \\
13 \\
8 \\
2 \\
1 \\
5 \\
5 \\
1 \\
1 \\
1 \\
1 \\
9\end{array}$ \\
\hline Family history of suicide (\%) & $3(6.7)$ & $6(11.3)$ & $16(34.0)$ \\
\hline Prescribed medications (\%) & $5(11.1)$ & $16(30.2)$ & $17(36.2)$ \\
\hline
\end{tabular}

* = Participants were asked to provide details of any current diagnosed medical conditions; physical and/or psychiatric/psychological $;{ }^{+}=$From Self-Injurious Thoughts and Behaviors Interview 
Table 2. Descriptive statistics (means and standard deviations) for main study variables in control, ideator and attempter groups $(n=145)$

\begin{tabular}{l|cccccccc}
\hline & \multicolumn{2}{c}{\begin{tabular}{c} 
Control \\
group \\
\cline { 2 - 9 }
\end{tabular}} & \multicolumn{2}{c}{$\begin{array}{c}\text { Ideator } \\
\text { group }\end{array}$} & \multicolumn{2}{c}{$\begin{array}{c}\text { Attempter } \\
\text { group } \\
(\mathrm{n}=53)\end{array}$} & $\begin{array}{c}\text { Ideator/Attempt } \\
(\mathrm{n}=47)\end{array}$ & $\begin{array}{c}\text { Combined } \\
(\mathrm{n}=100)\end{array}$ \\
\cline { 2 - 9 } & $\boldsymbol{M}$ & $\boldsymbol{S D}$ & $\boldsymbol{M}$ & $\boldsymbol{S D}$ & $\boldsymbol{M}$ & $\boldsymbol{S D}$ & $\boldsymbol{M}$ & $\boldsymbol{S D}$ \\
\hline Resting cortisol (nmol/L) & 3.99 & 2.26 & 4.49 & 3.17 & 4.02 & 3.34 & 4.26 & 3.26 \\
AUCg (nmol/L) & 32.49 & 23.07 & 29.97 & 22.93 & 23.52 & 17.30 & 26.75 & 20.12 \\
AUCi (nmol/L) & 10.05 & 20.04 & 9.34 & 16.65 & 3.40 & 10.60 & 6.37 & 13.63 \\
Physical neglect & 6.42 & 2.34 & 7.04 & 2.66 & 9.36 & 3.96 & 8.20 & 3.31 \\
Emotional abuse & 7.20 & 2.86 & 9.64 & 4.55 & 15.15 & 6.20 & 12.40 & 5.38 \\
Emotional neglect & 8.67 & 4.04 & 11.04 & 4.64 & 15.81 & 5.91 & 13.43 & 5.28 \\
Physical abuse & 5.49 & 1.16 & 6.34 & 2.36 & 9.29 & 5.53 & 7.82 & 3.95 \\
Sexual abuse & 5.58 & 3.03 & 6.36 & 3.86 & 9.14 & 6.10 & 7.75 & 4.98 \\
Total CTQ exposure & 0.27 & 0.65 & 0.68 & 1.07 & 2.30 & 1.71 & 1.49 & 1.39 \\
Beck Depression Inventory & 8.35 & 7.93 & 22.33 & 12.07 & 23.12 & 12.04 & 22.73 & 12.06 \\
Beck Hopelessness Scale & 3.64 & 3.23 & 10.41 & 5.01 & 10.02 & 6.30 & 10.22 & 5.66 \\
\hline
\end{tabular}

Note: $\mathrm{AUCg}=$ area under the curve with respect to ground; $\mathrm{AUCi}=$ area under the curve with respect to increase; Total CTQ = total Childhood Trauma Questionnaire exposure score (moderate or severe) 
Figure 1. Childhood trauma scores in attempter, ideator and control groups $(n=145)$

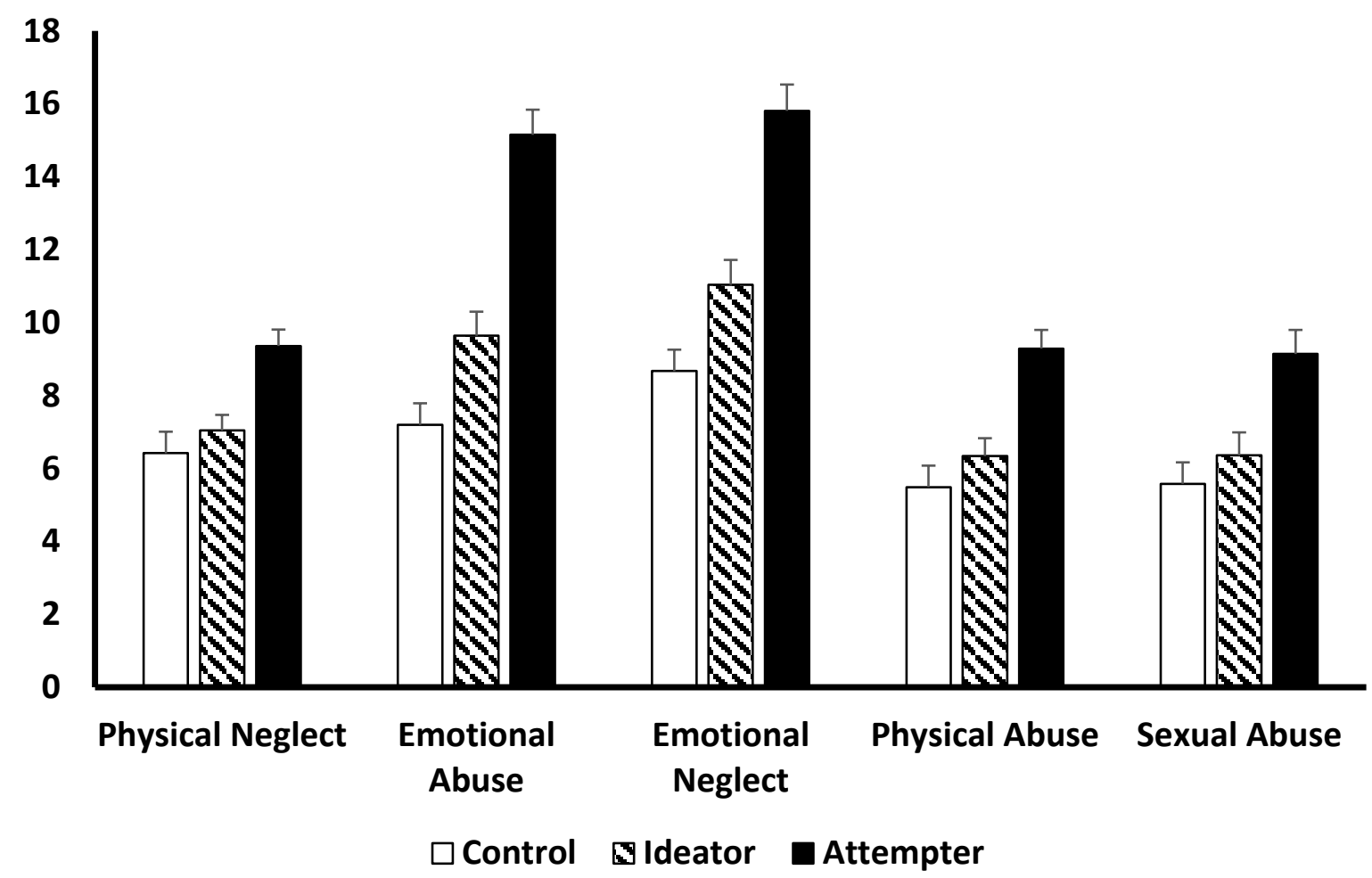

Figure 2: Total exposure to any type of "Moderate to Severe" childhood trauma $(n=100)$

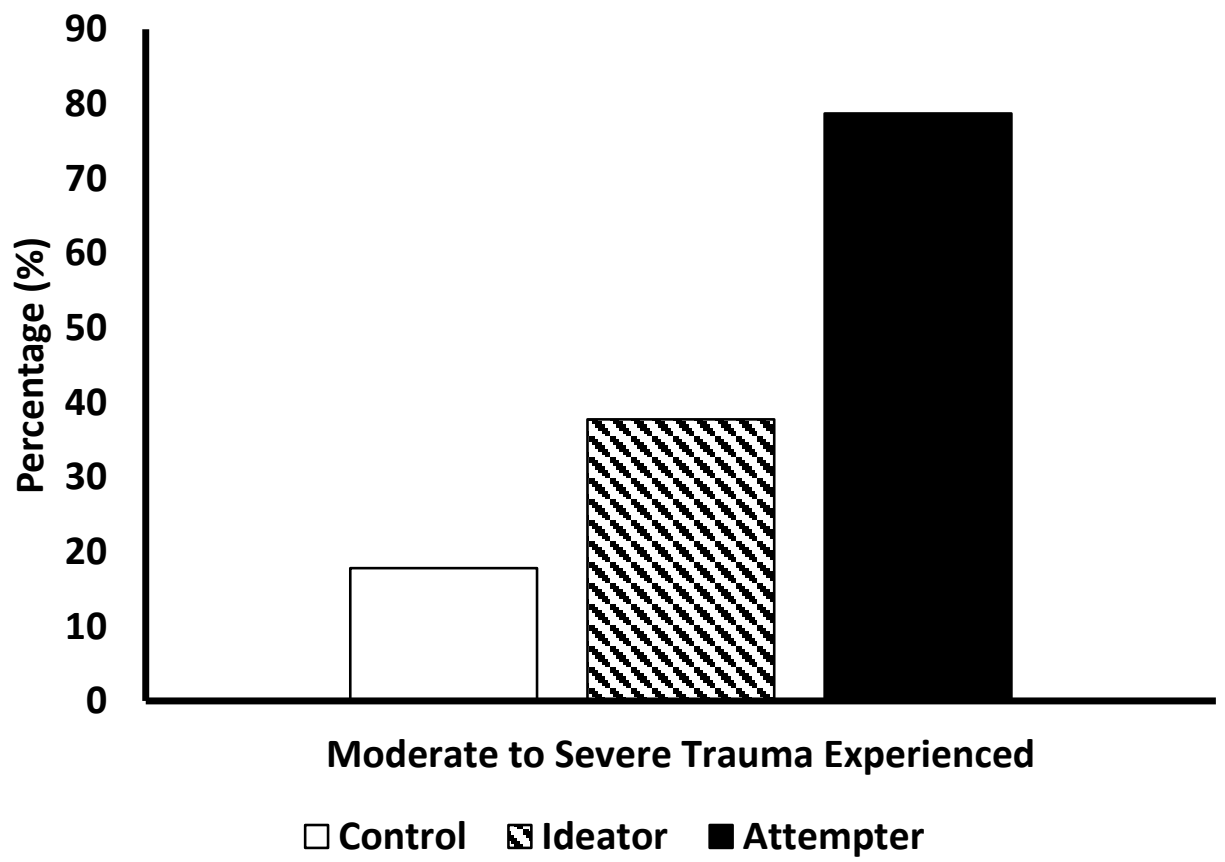


Table 3. Hierarchical regression analyses testing the effects of childhood trauma on resting cortisol levels in suicide attempters and ideators $(n=100)$

\begin{tabular}{|c|c|c|c|c|c|c|}
\hline & & $\begin{array}{c}\beta \\
\text { Step } 1\end{array}$ & $\begin{array}{c}\beta \\
\text { Step } 2\end{array}$ & $\begin{array}{c}\beta \\
\text { Step } 3\end{array}$ & $\begin{array}{c}\Delta \mathbf{R}^{2} \text { for } \\
\text { step }\end{array}$ & $\begin{array}{c}\text { Total } \\
\mathbf{R}^{2}\end{array}$ \\
\hline \multicolumn{7}{|c|}{$\begin{array}{l}\text { Resting } \\
\text { cortisol }\end{array}$} \\
\hline \multirow[t]{6}{*}{ Step 1} & Time of day & $-0.20 *$ & $-0.22 *$ & $-0.23^{*}$ & 0.09 & \\
\hline & Age & -0.02 & 0.03 & -0.01 & & \\
\hline & Gender & -0.19 & -0.18 & -0.20 & & \\
\hline & BMI & 0.11 & 0.11 & 0.11 & & \\
\hline & Medication usage & 0.10 & 0.13 & 0.12 & & \\
\hline & Smoking status & -0.06 & -0.05 & -0.09 & & \\
\hline Step 2 & Childhood trauma & & $-0.24 *$ & $-0.22 *$ & $0.05^{*}$ & \\
\hline \multirow[t]{2}{*}{ Step 3} & Depression & & & 0.14 & & \\
\hline & Hopelessness & & & $-0.26^{*}$ & 0.04 & 0.18 \\
\hline
\end{tabular}

Note: $*=p<0.05$ 
Table 4. Hierarchical regression analyses testing the effects of childhood trauma on cortisol reactivity to stress (AUCg and $\mathrm{AUCi}$ ) in suicide attempters and ideators $(\mathrm{n}=100)$

\begin{tabular}{|c|c|c|c|c|c|c|}
\hline & & $\begin{array}{c}\beta \\
\text { Step } 1\end{array}$ & $\begin{array}{c}\beta \\
\text { Step } 2\end{array}$ & $\begin{array}{c}\beta \\
\text { Step } 3\end{array}$ & $\begin{array}{c}\Delta R^{2} \text { for } \\
\text { step }\end{array}$ & $\begin{array}{c}\text { Total } \\
\mathbf{R}^{2}\end{array}$ \\
\hline \multicolumn{7}{|l|}{ AUCg } \\
\hline \multirow[t]{6}{*}{ Step 1} & Time of day & -0.10 & -0.12 & -0.14 & $0.13^{*}$ & \\
\hline & Age & -0.07 & -0.01 & -0.02 & & \\
\hline & Gender & $-0.24 *$ & $-0.23 *$ & $-0.24 *$ & & \\
\hline & BMI & 0.05 & 0.05 & 0.06 & & \\
\hline & Medication usage & $0.24 *$ & $0.27 * *$ & $0.27 * *$ & & \\
\hline & Smoking status & -0.11 & -0.11 & -0.12 & & \\
\hline Step 2 & Childhood trauma & & $-0.23 *$ & $-0.22 *$ & $0.05^{*}$ & \\
\hline \multirow[t]{2}{*}{ Step 3} & Depression & & & 0.06 & & \\
\hline & Hopelessness & & & -0.09 & 0.02 & 0.20 \\
\hline \multicolumn{7}{|l|}{$\mathbf{A U C i}$} \\
\hline \multirow[t]{6}{*}{ Step 1} & Time of day & 0.06 & 0.06 & 0.05 & 0.07 & \\
\hline & Age & 0.04 & 0.05 & 0.08 & & \\
\hline & Gender & -0.04 & -0.03 & -0.03 & & \\
\hline & BMI & -0.04 & -0.04 & -0.03 & & \\
\hline & Medication usage & 0.20 & 0.21 & $0.22 *$ & & \\
\hline & Smoking status & -0.12 & 0.12 & -0.10 & & \\
\hline Step 2 & Childhood trauma & & -0.05 & -0.05 & 0.00 & \\
\hline \multirow[t]{2}{*}{ Step 3} & Depression & & & -0.18 & & \\
\hline & Hopelessness & & & -0.12 & 0.02 & 0.09 \\
\hline
\end{tabular}

Note: $*=p<0.05 ; *=p<0.01$ 
Figure 3. Effects of childhood trauma levels on cortisol reactivity to stress in combined group of suicide attempters and ideators $(n=100)$

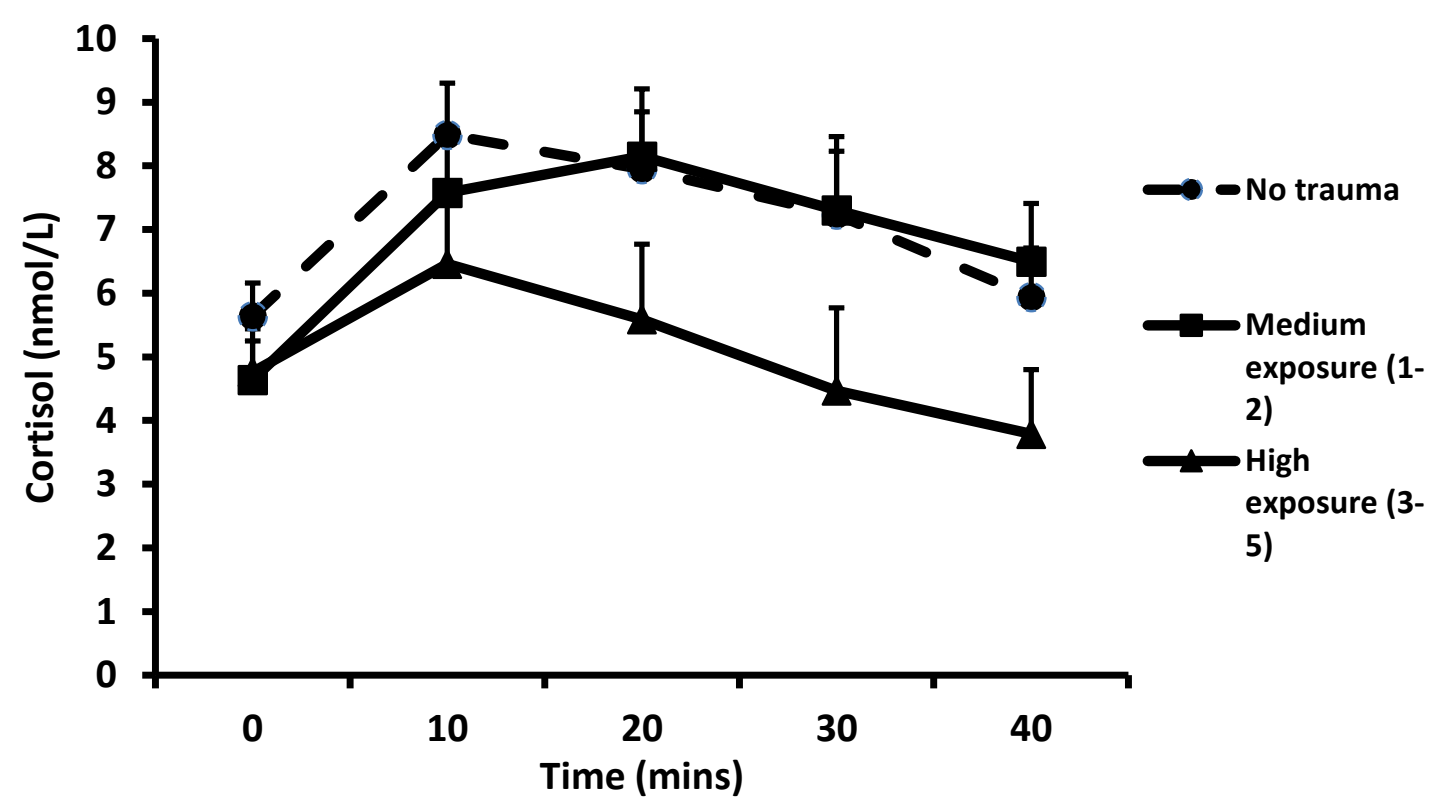

